Apuntes Universitarios, 2022: 12 (2), abril-junio

ISSN: 2304-0335 DOI: https://doi.org/10.17162/au.v12i2.1038

\title{
Clima social familiar en la autoestima de estudiantes del sudeste de la Amazonía peruana
}

\section{Family social climate on self-esteem of students in the southeastern Peruvian Amazon}

\author{
Jimmy Nelson Paricahua Peralta, ${ }^{1 \mathrm{a}}$ Thony Abel Lazo Herrera,${ }^{2}$ Braulio Pedro Espinoza Flores, ${ }^{3}$ \\ Libertad Velázquez Giersch ${ }^{4}$
}
Universidad Nacional Amazónica de Madre de Dios, Perú ${ }^{12}$
Universidad Nacional del Callao, Lima, Perú ${ }^{3}$
Universidad César Vallejo, Lima, perú ${ }^{4}$

(iD) ORCID ID: https://orcid.org/0000-0001-9399-5956 1

ORCID ID: https://orcid.org/0000-0003-1497-4546²

ORCID ID: https://orcid.org/0000-0002-5855-9750³

ORCID ID: https://orcid.org/0000-0001-8608-269X

Recibido: 03 de junio de 2021

Aceptado: 23 de diciembre de 2021

\section{Resumen}

El presente estudio tuvo como objetivo establecer la relación que tiene el clima social familiar con la autoestima de los estudiantes de una institución educativa de la ciudad de Puerto Maldonado, Perú. El enfoque fue cuantitativo, el diseño no experimental, el tipo, descriptivo correlacional y la muestra estuvo conformada por 246 estudiantes de educación secundaria a quienes se les aplicó la Escala de Clima Social Familiar y el Inventario de Autoestima. Los resultados indican que los estudiantes se caracterizaban por percibir que el clima social familiar tenía una tendencia desfavorable y tenían niveles medios de autoestima. Del mismo modo, se determinó que el coeficiente de correlación rho de Spearman entre las variables clima social familiar y la autoestima, según la percepción de los estudiantes, fue de 0,678 con un p-valor inferior al nivel de significancia $(\mathrm{p}<0,05)$, lo cual indica que existía una relación directa entre ambas variables. Por último, se determinó que la variable autoestima se relacionaba de manera directa con las dimensiones relación familiar $(\mathrm{rho}=0,541 ; \mathrm{p}=0,05)$, desarrollo familiar $(\mathrm{rho}=0,590 ; \mathrm{p}=0,05)$ y estabilidad familiar $(\mathrm{rho}=$ $0,612 ; p=0,05)$. Se concluyó que es necesario la implementación de estrategias de socialización familiar, potenciando sus fortalezas como la autoestima acorde a sus actitudes naturales, teniendo como unidad a la familia que, bajo su concepción perceptual como un cimiento de fortaleza a desarrollar.

Palabras clave: Psicología de la educación, familia, estudiante, relaciones interpersonales, Perú.

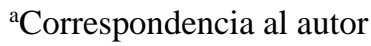

E-mail: jparicahua@unamad.edu.pe 


\begin{abstract}
The objective of the study was to establish the relationship that the family social climate has with the self-esteem of the students of an educational institution in the city of Puerto Maldonado, Peru. The approach was quantitative, non-experimental design, type, descriptive, correlational, and the sample was made up of 246 secondary school students to whom the Family Social Climate Scale and the Self-Esteem Inventory were applied. The results indicate that the students were characterized by perceiving that the family social climate had an unfavorable tendency and had average levels of self-esteem. Similarly, it was determined that the Spearman rho correlation coefficient between the variables, family social climate and self-esteem, according to the students' perception, was 0.678 with a p-value below the significance level $(\mathrm{p}<0.05)$, which indicates that there was a direct relationship between both variables. Finally, it was determined that the selfesteem variable was directly related to the dimensions of family relationship $(r h o=0.541 ; p=0.05)$, family development $(\mathrm{rho}=0.590 ; \mathrm{p}=0.05)$ and family stability $(\mathrm{rho}=0.612 ; \mathrm{p}=0.05)$. It was concluded that it is necessary to implement family socialization strategies, enhancing their strengths such as self-esteem according to their natural attitudes, having the family as a unit, which, under its perceptual conception, as a foundation of strength to be developed.
\end{abstract}

Keywords: Psychology of education, family, student and interpersonal relationships, Peru.

\title{
Introducción
}

Actualmente, las circunstancias generadas por la globalización han impactado y modificado la organización familiar, originando un estilo de vida cambiante, fundando en aspectos competenciales, que afectan la estructura de valores y la autoestima de los adolescentes, condicionándolos a convivir y comunicarse de una manera diferente, esta presión exige la búsqueda de nuevos modelos para sobrellevar las tensiones ocasionadas por el medio y alcanzar el éxito (Navarrete \& Ossa, 2013).

Por otro lado, la gran parte de los adolescentes está marcada por el desorden en las emociones, claros ejemplos son: la fábula personal, de invencibilidad y la audiencia imaginaria, ocasionados por aspectos madurativos y las limitaciones del individuo cuando realiza $\mathrm{o}$ experimenta nuevas acciones o actividades que le producen sensaciones diferentes, cambios en el desenvolvimiento personal y en las relaciones con individuos del entorno, determinadas por la autoestima (Llanca-Sánchez \& Armas-Zambrano, 2020).

De acuerdo a Díaz et al. (2018), los diferentes estilos educativos intervienen en el desarrollo de la adolescencia como un estado limitante en el fortalecimiento y el desarrollo de la autoestima, debido a que es el periodo de establecimiento de la identidad de la persona como un ser social, en el que el adolescente identifica sus limitaciones, posibilidades, talentos, desventajas y 
oportunidades que condicionan su forma de actuar en la práctica social, por lo que la familia como la escuela cumplen un rol determinante en su fortalecimiento.

Entonces, es indudable la importancia de la familia como un sistema social conformada por una serie de influencias y relaciones recíprocas entre sus miembros, destinadas a fomentar y fortalecer el desarrollo de capacidades emocionales, afectivas y cognitivas, dentro de un clima social, enriquecido por estímulos comunicativos, de cooperación mutua, compromiso y de crecimiento personal (Pi \& Cobián, 2016).

Por su parte, Moss (1974) define al clima social familiar como la percepción de las cualidades socioambientales que presenta una familia, la cual se describe de acuerdo a la forma cómo se interrelacionan sus integrantes, los factores del desarrollo que presentan más relevancia en ella y su estructura. Otros autores señalan que es el conjunto de aportes personales de cada uno de los integrantes de la familia, la cual se traduce en algo que brinda emoción (Zimmer y Locke, 2007). Así pues, el clima es más adecuado a medida que se fortalecen las relaciones entre sus integrantes.

De otro ángulo, Moya y Cunza (2019), consideran como criterios relacionados al clima social familiar a las relaciones interpersonales, el desarrollo personal y la estabilidad. Estas dimensiones se consolidan a partir de condiciones, actitudes y comportamientos de los integrantes del grupo familiar y serán positivas cuando se produzca la integración de sus miembros, de esta manera, las relaciones estarían determinadas por el nivel de comunicación, la libertad de expresión que se fomenta en el hogar y posiblemente se verán afectadas por los conflictos, su duración y continuidad (Reyes et al., 2019).

La familia representa un entorno de práctica y perfeccionamiento en la personalidad que regirá al adolescente, es el ambiente, el agente socializador que prepara, entrena y perfecciona la forma de actuar de los miembros, por tanto, los recursos afectivos, comunicativos, las experiencias, casos, ejemplos descritos y explicados son determinantes en su formación (De La Villa y Ovejero, 2015).

Asimismo, la labor educativa es condicionante, debido a que, en la práctica la autoestima representa una de las fuentes principales para que se origine y mantenga la motivación, porque involucra bases elementales como la autoeficacia, autoconcepto y la autorrealización que determinan la personalidad, así como la vida emocional y afectiva de los adolescentes (Silva \& Mejía, 2015). Así pues, las diferencias en la valoración que hacen los estudiantes de sí mismos 
afectan al nivel de autoestima y tendría como consecuencia determinadas situaciones socioeconómicas específicas, actuando como modeladores de las respuestas sociales (Tabernero, Serrano \& Mérida, 2017). Cabe indicar, la autoestima normal y elevada es un factor protector ante la intensidad de los problemas socio-familiares y el incremento de diversos factores de riesgo psicosocial (Andrade et al., 2017).

A la luz de este contexto, la presente investigación será relevante ya que se podrá conocer cómo los estudiantes perciben el entorno familiar y las interacciones que se producen en él, qué niveles de autoestima presentan y cómo se relacionan ambas variables. En base a los hallazgos, el equipo directivo de la institución educativa podría gestionar la presencia de especialistas para que desarrollen talleres y charlas donde promuevan la reflexión en los padres de familia sobre la importancia de mantener una dinámica familiar favorable, donde las interrelaciones se basen en el respeto, la responsabilidad, la solidaridad y empatía. Por otra parte, los docentes, mediante el trabajo en tutoría, podrán desarrollar estrategias psicoeducativas para mejorar la autoestima de los estudiantes. Finalmente, el objetivo de la presente investigación fue establecer la relación que tiene el clima social familiar con la autoestima de los estudiantes de una institución educativa de la ciudad de Puerto Maldonado, Perú.

\section{Metodología}

\section{Diseño}

El enfoque de investigación fue cuantitativo, puesto que se realizó la recolección de datos para responder a las preguntas de investigación y contrastar las hipótesis definidas con antelación, haciendo uso de la estadística (Sánchez et al., 2020). Respecto al diseño, fue no experimental, puesto que las variables no fueron manipuladas, sino se observaron tal como se dieron en su entorno para luego analizarlas (Hernández \& Mendoza, 2018). En cuanto al tipo, fue descriptivo correlacional de corte transversal puesto que se describieron las propiedades y características de la variables, se determinó si se relacionaban de manera significativa y la recolección de datos fue en un solo momento, respectivamente (Bisquerra, 2009).

\section{Muestra}

La población fue constituida por 683 adolescentes que cursaban la educación secundaria en la Institución Educativa Aplicación Nuestra Señora del Rosario de la Ciudad de Puerto Maldonado 
mientras que la muestra fue constituida por 246 estudiantes, cantidad obtenida mediante un muestreo probabilístico con un nivel de confianza del 95\% y un nivel de significancia del $5 \%$.

\section{Instrumentos}

Se utilizó la técnica de la encuesta mientras que los instrumentos fueron la Escala de Clima Social Familiar y el Inventario de Autoestima de Rosenberg. La Escala de Clima Social Familiar fue diseñada por Moos y Tricket (1974) y adaptada al contexto peruano por Ruiz y Guerra (1993). Consta de 90 ítems de tipo dicotómico (verdadero y falso) distribuidos en tres dimensiones: relación familiar, desarrollo familiar y estabilidad familiar. Sus cualidades psicométricas se determinaron a través de los procedimientos de validez y confiabilidad. La validez de contenido fue determinada mediante la técnica de juicio de expertos. Para ello se recurrió a 3 especialistas, los cuales determinaron que la escala tenía una adecuada validez de contenido. Respecto a la confiabilidad, fue hallada a través de una prueba piloto al $20 \%$ de la muestra, obteniéndose un coeficiente Kuder-Richardson de 0,845 lo que significó que la escala poseía una adecuada consistencia interna.

Por otro lado, el Inventario de Autoestima fue elaborado por Rosenberg (1965), está conformado por 10 ítems de tipo Likert (muy de acuerdo, de acuerdo, en desacuerdo y muy en desacuerdo) distribuidos en 5 dimensiones: dimensión física, dimensión social, dimensión afectiva y dimensión académica. Sus propiedades psicométricas también fueron determinadas mediante los procedimientos previamente descritos de validez y confiabilidad, concluyéndose que posee una adecuada validez de contenido. Asimismo, el coeficiente Alfa de Cronbach fue de 0,854 lo que significó que el inventario era confiable.

\section{Aspectos éticos}

Para la recolección de datos, se pidió la autorización a las autoridades educativas y, una vez obtenida la misma, se determinó el número de secciones y la cantidad de estudiantes a encuestar. Luego, se remitió a los padres de familia y tutores una carta de consentimiento informado para que sus hijos o apoderados participen del estudio. Finalmente, se procedió a aplicar los instrumentos de manera grupal bajo el acompañamiento de los investigadores. 


\section{Procesamiento de datos}

Con el propósito de efectuar el análisis estadístico se utilizó el Software SPSS versión 22, donde se sistematizaron los resultados mediante figuras. Asimismo, el contraste de hipótesis fue realizado usando el estadístico rho de Spearman puesto que las variables tenían un nivel de medición ordinal y no cumplían con el supuesto de normalidad.

\section{Resultados}

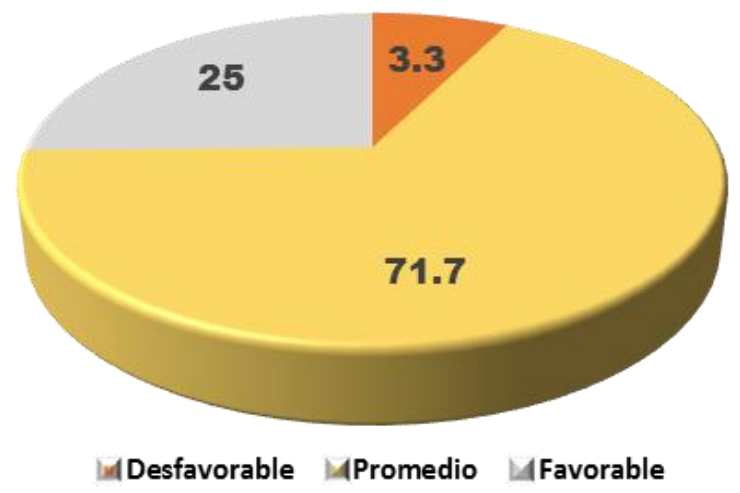

Figura 1. Resultados de la percepción del clima social familiar de los estudiantes

De acuerdo a la información analizada (Figura 1), se identificó que la percepción del clima social familiar de los estudiantes se ubica en el nivel promedio con el 71,67\%. Ello indica que las familias de los estudiantes están integradas, sin embargo, no logran consolidar en su totalidad un ambiente positivo de unión familiar necesario para desenvolverse y afrontar positivamente circunstancias cotidianas. El 25\% de la muestra está en el nivel favorable, lo que implica que la interacción existente entre los integrantes de cada familia de los estudiantes era positiva y de unión familiar, cualidades necesarias para expresar emociones, afrontar problemas, respetar las normas de convivencia y adecuarse a las situaciones de cambio. Por último, el nivel desfavorable cuenta con el 3,33\%, lo que significa que una pequeña proporción de las relaciones familiares que se dan entre los integrantes de los estudiantes tienen serias limitaciones debido al resquebrajamiento en la unión, integración y cohesión del núcleo familiar; limitante para convivir en un ambiente adecuado de aprendizaje y formación. 


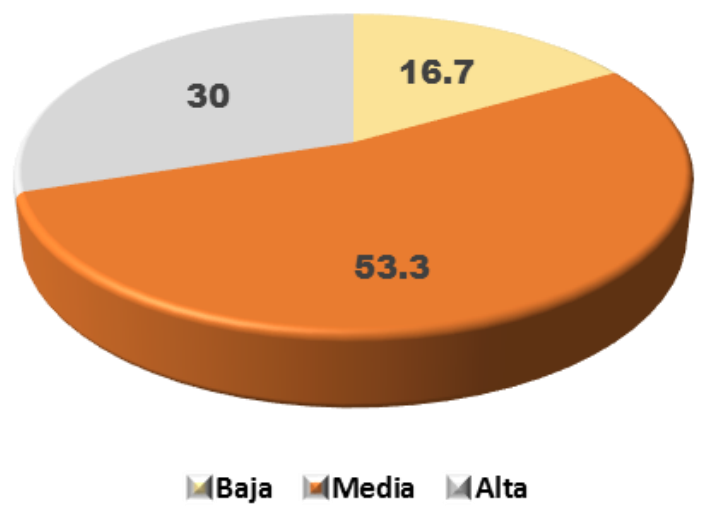

Figura 2. Resultados del nivel de autoestima de los estudiantes

Por otra parte, el 53,3\% de la muestra estudiada puntuó una autoestima en el nivel de medio, lo que significa que los estudiantes cuentan con un sentido positivo de aceptación a su condición física, sin embargo, para poder integrarse a los círculos sociales, así como, adaptarse a la vida académica necesitan del apoyo familiar, docente y demás integrantes de la institución educativa. Por otra parte, el $30 \%$ tiene una autoestima alta, lo que indica que los estudiantes tienen un sentimiento valorativo de aprecio hacia sí mismos que les permite tener estima a su condición física, integrarse en la sociedad, así como tener una autopercepción positiva de su vida académica. Por último, el 16,67\% posee una autoestima baja, por tanto, los estudiantes tienen dificultades para aceptarse a sí mismos, presentan problemas de integración en los círculos sociales, así como se les dificulta consolidar su vida académica.

\section{Tabla 1}

Correlación entre la variable clima social familiar y la autoestima de los estudiantes

\begin{tabular}{lllcc}
\hline & & & Clima social familiar & Autoestima \\
\hline & Clima social & Coeficiente de correlación & 1,000 &, $678^{* *}$ \\
& familiar & Sig. (bilateral) &. &, 000 \\
Rho de & & $\mathrm{N}$ & 246 & 246 \\
Spearman & & Coeficiente de correlación &, $678^{* *}$ & 1,000 \\
& \multirow{2}{*}{ Autoestima } & Sig. (bilateral) &, 000 &. \\
& & $\mathrm{~N}$ & 246 & 246 \\
\hline
\end{tabular}

**. La correlación es significativa en el nivel 0,01 (2 colas).

Fuente: Base de datos 
El coeficiente de correlación rho de Spearman entre las variables clima social familiar y la autoestima, según la percepción de los estudiantes, fue de 0,678 , lo cual indica la existencia de una correlación positiva moderada; asimismo, la significación asintótica fue de 0,000 , lo que indica que el p-valor fue menor al 0,05 ubicándose dentro del margen de error, por lo que se rechaza la hipótesis nula y se acepta la hipótesis alterna que existe relación entre las variables clima social familiar y autoestima.

\section{Tabla 2}

Coeficientes de determinación entre las variables y dimensiones del clima social familiar y la autoestima de los estudiantes

\begin{tabular}{lcc}
\hline \multicolumn{1}{c}{ Dimensiones } & Autoestima & Sig. Asintótica \\
\hline Relación familiar &, $541^{* *}$ &, 000 \\
Desarrollo familiar &, $590^{* *}$ &, 000 \\
Estabilidad familiar &, $612 * *$ &, 000 \\
\hline$* *$ La correlación es significativa en el nivel 0,01 (2 colas). \\
Fuente: Base de datos
\end{tabular}

El coeficiente de correlación rho de Spearman entre las dimensiones de la variable clima social familiar y la variable autoestima dieron como resultado que la dimensión relación familiar obtuvo 0,541 ; la segunda dimensión desarrollo familiar tuvo 0,590; por último, la dimensión estabilidad familiar fue de 0,612. Por otra parte, el p-valor fue menor a 0,05, por lo que se acepta la hipótesis que existe relación entre las dimensiones de la variable clima social familiar y la variable autoestima.

\section{Discusión}

El principal entorno social donde se desenvuelven los adolescentes es la familia (Bolaños \& Stuart, 2019). Se describe como el escenario en el que grupos definidos de personas funcionan e interactúan (Suárez \& Vélez, 2018). Entonces, es un lugar donde ellos socializan en el entorno familiar, aprenden la regulación de las emociones, la cooperación, la afirmación, la resolución de conflictos y las habilidades de comunicación (Flores et al., 2016). En ese sentido, el presente estudio tuvo como objetivo establecer la relación que tiene el clima social familiar con la autoestima de los estudiantes de una institución educativa de la ciudad de Puerto Maldonado, Perú.

Un primer hallazgo indica que la percepción del clima social familiar de la mayoría de estudiantes se ubicaba en el nivel promedio, es decir, las familias de los estudiantes estaban 
integradas, sin embargo, no lograban consolidar un ambiente positivo de unión familiar necesario para desenvolverse y afrontar positivamente circunstancias cotidianas. Asimismo, los estudiantes contaban con un sentido positivo de aceptación a su condición física para poder integrarse a los círculos sociales, sin embargo, para adaptarse a la vida académica necesitan del apoyo familiar, docente y demás integrantes de la institución educativa, por tanto, mientras más consolidado esté el clima social familiar, la autoestima será fortalecida. Lo expuesto sería explicado debido a que muchos de los padres de familia solían trabajar durante extensas jornadas al día para brindar el sustento económico familiar, por lo que en ocasiones descuidaban su rol protector, educativo y afectivo.

Los resultados obtenidos son corroborados por Martínez et al. (2013), quienes concluyeron que la forma de apoyo y la organización familiar a partir de un modelo adecuado colabora en el comportamiento y la conducta de sus integrantes, lo que se reflejará claramente en su vida y desenvolvimiento académico como social. Sin embargo, como advierte Gómez et al. (2013), las condiciones negativas en la dinámica de la familia conducen a la disfuncionalidad, afectando seriamente el desempeño académico. Esto se corrobora en la propuesta de Lastre et al. (2018), quien comprobó que el desempeño de los alumnos está determinado por las relaciones familiares.

Asimismo, Pinto (2016), quien resalta la importancia del núcleo familiar en la formación de valores y de principios enfocados en la cordialidad, el respeto y la integración. No obstante, como indica Sagbaicela (2018), los problemas de conducta y la poca resolución de los conflictos representan la problemática que se origina en el núcleo de las familias con disfunción. Por tanto, todo esto afectaría a los estudiantes como demuestran Rodríguez y Caño (2012), que las deficiencias de autoestima generan una serie de problemas de conducta, emocionales, así como, de salud. Por tanto, como indica De Tejada (2010), la autoestima no solo obedece a aspectos de tipo madurativo sino experiencial.

Otro hallazgo indica que el mayor porcentaje de estudiantes presentaban un nivel de autoestima medio, lo que significa que los estudiantes contaban con un sentido positivo de aceptación a su condición física, sin embargo, para poder integrarse a los círculos sociales así como adaptarse a la vida académica necesitaban del apoyo familiar, principalmente. La información descrita podría explicarse debido al poco apoyo familiar que existe, lo cual obstaculizaría que los estudiantes puedan sentirse valorados, queridos, respeten las reglas del hogar y desarrollen la resiliencia. 
El hallazgo expuesto coincide con lo reportado por Estrada et al. (2021) quienes determinaron que los estudiantes presentaban niveles moderados de desarrollo de su autoestima, es decir, ellos estaban consolidando su autoestima, no obstante, al atravesar una etapa de la vida caracterizada por la presencia de muchos factores de riesgo, era menester que sea desarrollada integralmente para que afronten positivamente las diversas situaciones adversas.

De acuerdo a Juan (2019), cuando la autoestima está comprometida en un nivel de bajo, existiría el alto pronóstico que afectaría a la persona para integrar o pertenecer a determinados grupos, ya sea en la escuela o la comunidad, como al auto conocimiento y la percepción de la propia identidad como un ser sociable; asimismo, será determinante la capacidad y el nivel que tengan los padres de familia para identificar, atender y orientar las dificultades que se presenten.

Respecto al resultado principal, se determinó que existía una relación positiva y moderada entre las variables clima social familiar y la autoestima de los estudiantes de una institución educativa de la ciudad de Puerto Maldonado, Perú. El coeficiente de correlación rho de Spearman fue de 0,678 y el p-valor fue inferior al nivel de significancia $(\mathrm{p}<0,05)$. El hallazgo expuesto coincide con diversos estudios (Llanca-Sánchez \& Armas-Zambrano, 2020; Povedano et al., 2011; Ferreyros, 2019; Krauss et al., 2020; Rezaei et al., 2015) donde se encontró que el clima social familiar se relacionaba de manera directa y significativa con la autoestima de los adolescentes.

De acuerdo a Ruiz y Carranza (2018), cuando la dinámica social de la familia es funcional y los estudiantes cuentan, sobre todo, con el apoyo y ayuda en la familia, se producirán las condiciones favorables para consolidar las relaciones entre los integrantes de la familia, así como el fortalecimiento y consolidación de la capacidad de afronte y resolución de problemas. Asimismo, la importancia de la familia como base del desarrollo permite consolidar en sus miembros otras capacidades relacionadas con las normas y los modelos de conducta, en especial, cuando están establecidos los roles que deban cumplir en la organización, este aspecto afianza la manera de involucrarse, en conjunto con las respuestas, el interés y el valor que le brinden a las actividades realizadas (Oliva \& Villa, 2014).

Del mismo modo, Sierra et al (2019) da la relevancia que tiene la composición familiar como base y núcleo para la formación personal de sus integrantes, la función representativa que cada uno de los miembros orientada hacia la formación de los valores, principios y el cumplimiento de normas, consolidado en la autovaloración, la atención y empeño que se ponga en las actividades 
que se realizan, al igual que en el rechazo o distanciamiento hacia el consumo de sustancias adictivas.

Cabe indicar que las diferentes actividades que afectan a la dinámica familiar están relacionadas con el estrato social al que pertenece la persona, debido a que existe la necesidad de obtener recursos económicos para el día a día, se dificulta la integración familiar por parte de sus miembros, ocasionando distanciamiento e independencia prematura que obliga a los integrantes de la familia tengan que aprender y consolidar por su propia cuenta una serie de criterios para su subsistencia en la sociedad, los cuales en muchos casos son negativos (Moreno et al., 2019).

Finalmente, es menester precisar que una de las limitaciones del presente estudio fue la cantidad de la muestra, que no permite realizar generalizaciones, restringiendo los hallazgos al presente grupo de estudiantes. Por ello, se recomienda que las futuras investigaciones aumenten la cantidad de la muestra e incluyan a estudiantes de otras instituciones educativas, tanto urbanas como rurales para que los resultados puedan generalizarse.

\section{Conclusión}

La implementación de estrategias para clima social familiar y la autoestima son tendencias en el desarrollo pedagógico que ha permitido empoderar a los estudiantes, contribuyendo en sus aprendizajes y conocimientos así como en su bienestar personal y familiar. Los hallazgos encontrados en la presente investigación permitieron concluir que existía una relación entre el clima social familiar y la autoestima de los estudiantes del sudeste de la Amazonía peruana. Del mismo modo, se halló que la variable autoestima se relacionaba con las dimensiones relación familiar, desarrollo familiar y estabilidad familiar. Por ello, se recomienda implementar estrategias de socialización familiar, potenciando sus fortalezas como la autoestima acorde a sus actitudes naturales, teniendo como unidad a la familia que, bajo su concepción perceptual, es un cimiento de fortaleza a desarrollar.

\section{Referencias}

Andrade, J., Duffay, L., Ortega, P., Ramirez, E. \& Carvajal, J. (2017). Autoestima y desesperanza en adolescentes de una institución educativa del Quindío. Duazary, 14 (2), 179-187. https://doi.org/10.21676/2389783X.1968

Bisquerra, R. (2009). Metodología de la Investigación Educativa. (2a ed.). Madrid: La Muralla S.A. 
Bolaños, D. \& Stuart, A. (2019). La familia y su influencia en la convivencia escolar. Revista $\begin{array}{llllll}\text { Universidad } & y & \text { Sociedad, } & 11 & \text { (5), }\end{array}$ https://rus.ucf.edu.cu/index.php/rus/article/view/1353

De La Villa, M. \& Ovejero, A. (2015). Relación entre el clima social familiar y las actitudes juveniles ante el acoso escolar. International Journal of Developmental and Educational Psychology, 5 (1), 329-342. https://doi.org/10.17060/ijodaep.2014.n1.v5.690

De Tejada, M. (2010). Evaluación de la autoestima en un grupo de escolares de la Gran Caracas. Liberabit, $16 \quad$ (1), 95-103. http://www.scielo.org.pe/scielo.php?pid=S172948272010000100010\&script=sci_abstract

Díaz, L., Fuentes, M. \& Senra, L. (2018). Adolescencia y autoestima: su desarrollo desde las instituciones educativas. Revista Conrado, $14 \quad$ (64), 98-103. https://conrado.ucf.edu.cu/index.php/conrado/article/view/793

Estrada, E., Gallegos, N., Mamani, H. \& Zuloaga, M. (2021). Autoestima y agresividad en estudiantes peruanos de educación secundaria. Archivos Venezolanos de Farmacología y Terapéutica, 40 (1), 81-87. http://saber.ucv.ve/ojs/index.php/rev_aavft/article/view/22350

Ferreyros, L. (2019). Comunicación familiar y autoestima en estudiantes de secundaria de la ciudad de Puno, Perú. $\quad$ Revista $\quad$ Espacios, $40 \quad$ 7-16. https://www.revistaespacios.com/a19v40n44/19404407.html

Flores, E., Garcia, M., Calsina, W. \& Yapuchura, A. (2016). Las habilidades sociales y la comunicación interpersonal de los estudiantes de la Universidad Nacional del Altiplano Puno.Comuni@cción: Revista de Investigación en Comunicación y Desarrollo, 7 (2), 5-14. https://www.comunicacionunap.com/index.php/rev/article/view/106

Gómez-, E., Castillo, I. \& Cogollo, Z. (2013). Predictores de disfunción familiar en adolescentes escolarizados Revista Colombiana de Psiquiatría, $42 \quad$ (1), 72-80. https://doi.org/10.1016/S0034-7450(14)60088-3

Hernández, R. \& Mendoza, C. (2018). Metodología de la investigación: las rutas cuantitativa, cualitativa y mixta. México: McGraw-Hill.

Juan, P. (2019). El juego divierte, forma, socializa y cura. Pediatría Atención Primaria, 31 (83), 307-312. $\quad$ https://scielo.isciii.es/scielo.php?script=sci_arttext\&pid=S1139$\underline{76322019000300022}$

Krauss, S., Orth, U. \& Robins, R. (2020). Family environment and self-esteem development: A longitudinal study from age 10 to 16. Journal of Personality and Social Psychology, 119 (2), 457-478. https://doi.org/10.1037/pspp0000263

Lastre, K., López, L. \& Alcázar, C. (2018). Relación entre apoyo familiar y el rendimiento académico en estudiantes colombianos de educación primaria. Psicogente, 21 (39), 102-115. http://doi.org/10.17081/psico.21.39.2825 
Llanca-Sánchez, B. B. \& Armas-Zambrano, N. (2020). Clima social familiar y autoconcepto en adolescentes de una institución educativa de Lima Norte. CASUS. Revista de Investigación y Casos En Salud, 5 (1), 26-33. https://doi.org/10.35626/CASUS.1.2020.245

Marín, M., Quintero, P. \& Rivera, S. (2019). Influencia de las relaciones familiares en la primera infancia. Poiésis, 10 (36), 164-183. https://doi.org/10.21501/16920945.3196

Martínez, G., Torres, M. \& Ríos, V. (2020). El contexto familiar y su vinculación con el rendimiento académico. IE Revista De Investigación Educativa De La REDIECH, 11, e657. https://doi.org/10.33010/ie_rie_rediech.v11i0.657

Moreno, J., Umbarila, L \& Franco, L. (2019). Relación entre dinámicas familiares, conductas externalizantes y autoestima en niños escolarizados. Psychologia: Avances de la Disciplina, 13 (2), 43-54. https://doi.org/10.21500/19002386.3954

Moss, R. (1974). Combined preliminary manual: Family work and group environment scales manual. California: Consulting Psychologist Press.

Moos, R. \& Trickett, E. (1974). Classroom environment scale manual. California: Ed. Consulting Psychologist Press.

Moya, M. \& Cunza, D. (2019). Clima social familiar y resiliencia en estudiantes de $3^{\circ}, 4^{\circ}$ y $5^{\circ}$ año de secundaria. Apuntes Universitarios, 9 (2), 73-82. https://doi.org/10.17162/au.v9i2.361

Navarrete, L., \& Ossa, C. (2013). Estilos parentales y calidad de vida familiar en adolescentes con conductas disruptivas. Ciencias Pedagógicas, $7 \quad$ (1), 47-56. http://www.scielo.edu.uy/scielo.php?pid=S1688-42212013000100005\&script=sci_arttext

Oliva, E. \& Villa, V. (2014). Hacia un concepto interdisciplinario de la familia en la globalización. Justicia Juris, 10 (1), 11-20. http://ojs.uac.edu.co/index.php/justicia-juris/article/view/295

Paricahua, J., Quispe, W. \& Ramos, D. (2016). Habilidades sociales, clima de aula y aprendizaje cooperativo de los estudiantes de las facultades de la Universidad Nacional Amazónica de $\begin{array}{lllll}\text { Madre de } & \text { Dios. }\end{array}$ https://journal.ceprosimad.com/index.php/ceprosimad/article/view/32

Pi, A. \& Cobián, A. (2016). Clima Familiar: una nueva mirada a sus dimensiones e interrelaciones. MULTIMED, $20 \quad$ 449-460. http://www.revmultimed.sld.cu/index.php/mtm/article/view/164/212

Pinto, R. (2016). La importancia de promover los valores del hogar hacia las escuelas primarias. Ra Xinhai, 12 (3), 271-283. http://www.revistas.unam.mx/index.php/rxm/article/view/71689

Povedano, A., Hendry, L., Ramos, M. \& Varela, R. (2011). Victimización escolar: clima familiar, autoestima y satisfacción con la vida desde una perspectiva de género. Psychosocial Intervention, 20 (1), 5-12. https://doi.org/10.5093/in2011v20n1a1

Reyes, V., Merino, T., Villavicencio, L., Mendoza, C. \& Sequera, A. (2019). El clima social familiar y la agresividad en educación primaria: un estudio de caso en Guayaquil - Ecuador. 
Research, Society and Development, 8 (11), 248111465. https://doi.org/10.33448/rsdv8i11.1465

Rezaei, A., Paki, S. \& Keshvari, M. (2015). The relationship between family functioning and selfesteem in female high school students of Isfahan, Iran, in 2013-2014. Iranian Journal of Nursing and Midwifery Research, $20 \quad$ 371-377. https://www.ncbi.nlm.nih.gov/pmc/articles/PMC4462064/

Ruiz, C. \& Guerra, E. (1993). Escala de Clima Social en la familia para Lima Metropolitana. Lima: Estandarización.

Ruiz P. \& Carranza R. (2018). Inteligencia emocional, género y clima familiar en adolescentes $\begin{array}{llllll}\text { peruanos. Acta Colombiana de Psicología, } 21 & \text { (2), }\end{array}$ https://doi.org/10.14718/ACP.2018.21.2.9

Rodríguez, C. \& Caño, A. (2012). Autoestima en la adolescencia: análisis y estrategias de intervención. International Journal of Psychology and Psychological Therapy, 12 (3), 389403. https://www.ijpsy.com/volumen12/num3/337/autoestimaen-la-adolescencia-anlisisES.pdf

Rosenberg, M. (1965). Society and the adolescent self-image. New Jersey: Princeton University Press.

Sagbaicela, J. (2018). La disfuncionalidad familiar y su incidencia en los problemas de aprendizaje de los niños de tercer año de educación general básica elemental. Espirales Revista Multidisciplinaria de investigación, 2 (20). https://doi.org/10.31876/re.v2i21.341

Sánchez, H., Reyes, C. \& Mejía, K. (2018). Manual de términos en investigación científica, tecnológica y humanística. Lima: Universidad Ricardo Palma.

Sierra, T., Sevilla, D. \& Martín, M. (2019). Profesor universitario, ser en resiliencia: una mirada a su labor en el contexto educativo actual. Diálogos sobre educación. Temas actuales en investigación educativa, 19 (10), 1-20. https://doi.org/10.32870/dse.vi19.539

Silva, I. \& Mejía, O. (2015). Autoestima, adolescencia y pedagogía. Revista Electrónica Educare, 19 (1), 241-256. http://dx.doi.org/10.15359/ree.19-1.13

Suárez, P. \& Vélez, M. (2018). El papel de la familia en el desarrollo social del niño: una mirada desde la afectividad, la comunicación familiar y estilos de educación parental. Psicoespacios, 12 (20), 173-198. https://doi.org/10.25057/21452776.1046

Tabernero, C., Serrano, A. \& Mérida, R. (2017). Estudio comparativo de la autoestima en escolares de diferente nivel socioeconómico. Psicología Educativa. Revista de los Psicólogos de la Educación, 23 (1), 9-17. https://doi.org/10.1016/j.pse.2017.02.001

Verdugo, J., Arguelles, J., Guzmán, J., Márquez, C., Montes, R. \& Uribe, I. (2014). Influencia del clima familiar en el proceso de adaptación social del adolescente. Psicología desde el Caribe, 31 (2), 207-222. https://doi.org/10.14482/psdc.31.2.6127 
Zimmer, M. \& Locke, E. (2007). The socialization of adolescent coping: Relationships at home and school. Journal of Adolescence. 39 1-16. https://doi.org/10.1016/j.adolescence.2005.03.001 